\title{
Correction: Role of novel histone modifications in cancer
}

\section{Muthu K. Shanmugam ${ }^{1}$, Frank Arfuso $^{2}$, Surendar Arumugam ${ }^{10}$, Arunachalam Chinnathambi ${ }^{3}$, Jinsong Bian ${ }^{1}$, Sudha Warrier ${ }^{4}$, Ling Zhi Wang ${ }^{1,5}$, Alan Prem Kumar $^{1,5,6,7,8}$, Kwang Seok Ahn ${ }^{9}$, Gautam Sethi ${ }^{1}$ and Manikandan Lakshmanan ${ }^{10,11}$}

${ }^{1}$ Department of Pharmacology, Yong Loo Lin School of Medicine, National University of Singapore, Singapore, Singapore

2 Stem Cell and Cancer Biology Laboratory, School of Biomedical Sciences, Curtin Health Innovation Research Institute, Curtin University, Perth, WA, Australia

${ }^{3}$ Department of Botany and Microbiology, College of Science, King Saud University, Riyadh, Kingdom of Saudi Arabia

${ }^{4}$ Division of Cancer Stem Cells and Cardiovascular Regeneration, School of Regenerative Medicine, Manipal Academy of Higher Education (MAHE), Bangalore, India

${ }^{5}$ Cancer Science Institute of Singapore, National University of Singapore, Singapore, Singapore

${ }^{6}$ Curtin Medical School, Faculty of Health Sciences, Curtin University, Perth, WA, Australia

${ }^{7}$ National University Cancer Institute, National University Health System, Singapore, Singapore

${ }^{8}$ Department of Biological Sciences, University of North Texas, Denton, Texas, USA

${ }^{9}$ College of Korean Medicine, Kyung Hee University, Dongdaemun-gu, Seoul, Korea

${ }^{10}$ Institute of Molecular and Cell Biology, A*STAR, Biopolis Drive, Proteos, Singapore, Singapore

11 Department of Pathology, National University Hospital Singapore, Singapore, Singapore

Published: April 10, 2018

Copyright: Shanmugam et al. This is an open-access article distributed under the terms of the Creative Commons Attribution License 3.0 (CC BY 3.0), which permits unrestricted use, distribution, and reproduction in any medium, provided the original author and source are credited.

This article has been corrected: The correct author name is given below:

\section{Jinsong Bian}

Original article: Oncotarget. 2018; 9:11414-11426. https://doi.org/10.18632/oncotarget.23356 